\title{
Le Millennium Ecosystem Assessment : anatomie d'une évaluation environnementale globale
}

\author{
Denis Pesche \\ Sociologue, CIRAD, UMR 5281 ART-Dev, 34398 Montpellier cedex 5, France
}

Comment se diffusent à l'échelle mondiale les représentations de la biodiversité ? Comment expliquer que la notion de services environnementaux, à la piètre définition scientifique, ait pu connaître un tel succès dans sa version économique de paiements pour services écosystémiques? Et cela aussi bien auprès des institutions internationales, des porteurs de projets et des populations qui pensent y trouver une rémunération de leurs efforts selon un parfait modèle gagnant-gagnant fondé sur le marché. L'article de Denis Pesche s'appuie sur la notion de configuration épistémique pour analyser la logique et les réseaux d'acteurs qui ont présidé à la publication du Millennium Ecosystem Assessment qui, tant par les procédures de sa conception que par son contenu, a constitué une étape-clé dans la construction et la diffusion d'une notion permettant d'expliciter les différentes valeurs que l'on peut donner à la biodiversité.

La Rédaction

\section{Mots-clés :}

environnement ; biodiversité ; évaluation globale environnementale ; service écosystémique ; sociologie
Résumé - La notion de service écosystémique (SE) a été élaborée progressivement dans les milieux scientifiques dans le courant des années 1990. C'est à l'occasion d'une évaluation environnementale globale, le Millennium Ecosystem Assessment (MA), que cette notion acquiert une reconnaissance internationale auprès d'un public diversifié (décideurs publics, secteur privé, ONG, etc.). Depuis 2005, la notion de SE connaît un fort développement dans différentes arènes : biodiversité, politiques sectorielles (forêts, agriculture, eau, etc.), changement global, etc. Cet article se fonde sur l'hypothèse exploratoire que le processus du MA, en s'appuyant sur l'articulation entre des réseaux diversifiés de scientifiques et de décideurs, a joué un rôle-clé dans la reconnaissance puis la large diffusion de cette notion de SE à différentes échelles (conventions internationales, politiques domestiques, dispositifs d'action publique locaux $)^{1}$. Il souligne aussi la place déterminante des acteurs insérés ou liés au champ scientifique des États-Unis.

\section{Keywords:}

environment;

biodiversity; global environmental assessment; ecosystem services; sociology

\begin{abstract}
The Millennium Ecosystem Assessment: anatomy of a global environmental assessment. The topic of ecosystem services (ES) has recently become a key reference for international environmental policies (broadly including forest policy, agro-environmental measures, conservation policies, etc.) This concept has been developed gradually in scientific circles in the course of the 1990s. It acquired international recognition with a varied public (policy makers, private sector, NGOs, etc.) during the Millennium Ecosystem Assessment (MA), a global environmental assessment. Since 2005, this notion is experiencing strong growth in different fields such as biodiversity, domestic policies (forestry, agriculture, water, etc.), global change, etc. Our paper is based on the exploratory assumption that the MA process, by organizing the relationship between various networks of scientists and policymakers, has played a key role in the launching and wide dissemination of the ES concept at different scales: international level, domestic policies, local public action mechanisms... It also emphasizes the decisive role played by actors involved in or related to the scientific field in the United States.
\end{abstract}

\footnotetext{
Auteur correspondant : denis.pesche@cirad.fr

1 Cet article est un des résultats du programme Serena, soutenu par le programme Systerra de l'ANR (ANR 08 STRA 13). Voir www.serena-anr.org.
} 


\section{Introduction}

Le Millenium Ecosystem Assessment (MA) fait référence dans le domaine de la biodiversité et des services écosystémiques. Cette évaluation environnementale globale réalisée entre 2001 et 2005 a joué un rôle central dans le processus de reconnaissance internationale de cette notion de service écosystémique (SE), comme en témoigne la croissance exponentielle des références à cette notion dans la littérature scientifique depuis 2006 (Méral, 2012). Cette reconnaissance s'est traduite en particulier par l'intégration de la notion de SE dans les textes de plusieurs conventions internationales et la mise en place de l'IPBES (Intergovernmental Science-Policy Platform on Biodiversity and Ecosystem Services). Cet article n'a pas pour objectif de discuter le cadre d'analyse et les concepts produits par le MA, exercice réalisé par d'autres publications (Carpenter et al., 2009 ; GómezBaggethun et al., 2010 ; Leemans, 2008 ; Lévêque, 2007 ; Norgaard, 2010). Il s'agit principalement de chercher à mieux comprendre les ressorts de la large et rapide diffusion de la notion de SE depuis 2006.

La littérature analysant les évaluations environnementales globales (Clark et al., 2006) estime que ces exercices internationaux mobilisant de nombreux chercheurs et experts ont de l'influence sur les décideurs et produisent des effets autant par la nature du processus lui-même que du fait des idées ou concepts qu'ils formulent. Pour ces auteurs, l'influence d'une évaluation environnementale globale repose sur les caractéristiques et l'étendue du processus pendant l'évaluation, mais aussi après la publication du rapport. La nature des réseaux d'experts, leurs interconnexions et les dispositifs permettant $d^{\prime}$ articuler divers types de connaissances dans une dynamique de dialogue entre experts, mais aussi entre les experts et les décideurs pendant l'évaluation, constituent un des apports de ce type d'exercice (Norgaard, 2008).

L'hypothèse exploratoire que nous formulons dans cet article est que la dynamique de réseau entre scientifiques, experts et décideurs, qui s'est construite avant et pendant le MA, a joué un rôle important dans l'écho rencontré par cette évaluation après 2005. Plus précisément, nous montrons que le MA a mobilisé une expertise diversifiée, puisant ses compétences et sa légitimité dans des univers relativement différents, et que la mise en réseau de ces compétences et «mondes » diversifiés a joué un rôle-clé dans l'écho rencontré par la notion de SE suite à la publication du MA en 2005.

La notion de « communauté épistémique » a été introduite par P.M. Haas afin d'examiner le rôle des réseaux d'experts basés sur le savoir dans l'analyse et la recherche de solutions à des problèmes complexes au niveau international (Haas, 1992, 2000). Pour Haas, ces réseaux jouent un rôle pour accompagner les États dans l'identification de leurs intérêts, cadrer les débats collectifs, proposer des politiques spécifiques et identifier les points saillants dans les négociations. La notion de «communauté épistémique» permet de repérer un réseau de professionnels à l'expertise et aux compétences reconnues dans un domaine particulier, et qui fait autorité par les connaissances «pertinentes » qu'il peut mobiliser pour les politiques dans ce domaine. Haas précise que la notion a été utilisée de différente manière mais souvent pour désigner des communautés scientifiques.

Un usage trop réifiant de cette notion souple de communauté épistémique peut induire deux risques. Le premier risque consiste à sous-évaluer les ancrages nationaux de ces configurations internationales: pour Y. Dezalay et B.G. Garth, « il n'est guère aisé d'échapper au courant dominant qui aboutit à isoler les stratégies internationales de leur contexte national, en les inscrivant dans des catégories à prétentions universelles, comme celles de "communautés épistémiques" ou de "réseaux d'activisme juridique" (advocacy networks) qui servent de panacée pour décrire, de manière aussi idéaliste que normative, l'émergence d'une "société civile internationale" [ ... car] cette approche se borne à souligner le caractère international des opérateurs, en négligeant les ressources et les choix stratégiques qu'ils doivent aux positions qu'ils occupent dans leur champ national » (Dezalay et Garth, 2002). Cette critique prend tout son sens dans le besoin de saisir les dimensions et racines sociologiques des ressources que les personnes engagées dans une "communauté épistémique » peuvent mobiliser, sachant qu'elles procèdent pour la plupart des espaces nationaux et ne se limitent pas forcément aux relations avec les sphères gouvernementales. Les ancrages nationaux dans les sociétés savantes, les organisations scientifiques ou les ONG peuvent jouer un rôle important comme nous le verrons dans la suite de l'article. Le second risque consiste à accorder trop de sens à la notion de communauté, induisant l'idée que ces « communautés» sont des groupes relativement homogènes qui permettent la promotion d'idées au niveau international. Ce risque est cependant mineur car P.M. Haas prévient justement du caractère possiblement hétérogène des communautés épistémiques et de la diversité possible de ses membres. Des travaux ultérieurs ont aussi montré la pertinence de la notion pour caractériser des regroupements relativement hétérogènes d'acteurs autour d'une idée ou d'un modèle technologique, comme la question de la séquestration du carbone (Stephens et al., 2011). Le caractère hétérogène et multiforme des réseaux engagés dans les évaluations environnementales globales a aussi été souligné par l'usage de la notion de "réseaux distribués " de recherche, d'évaluation et de décision. Ces systèmes d'acteurs se caractérisent par une organisation polycentrique, le 
rôle important des médiateurs et des organisationsfrontières, et des modes d'organisation adaptatifs (Cash et Clark, 2001).

Dans le travail de recherche dont cet article rend compte, nous avons forgé la notion de « configuration épistémique », inspirée de celle de communauté épistémique, mais s'en distinguant par l'idée de souligner le caractère composite et relativement instable des collectifs qui se sont constitués à l'occasion du MA (Pesche et al., 2012). La notion de configuration épistémique, à caractère principalement exploratoire, permet de saisir le travail de mise en réseau de sous-ensembles composés d'acteurs diversifiés par leurs appartenances institutionnelles, leur nationalité, leurs trajectoires scientifiques, etc. En analysant la structure et les articulations entre ces différents réseaux, il s'agira de saisir la dynamique de construction de la notion de SE et les canaux de sa diffusion ultérieure, principal "véhicule» des effets du MA.

Nous présentons rapidement dans une première section les mécanismes organisationnels du MA qui articulent étroitement des scientifiques et des décideurs, et ce, tout au long des grandes étapes du MA. La nature profondément imbriquée du processus s'exprime aussi et surtout du fait de la trajectoire des personnes impliquées de près dans la conduite du MA. Un nombre limité d'individus est à l'origine de la construction d'une configuration épistémique, étudiée dans la deuxième section, qui est parvenue à connecter quatre sous-réseaux distincts. La diversité des réseaux d'acteurs mobilisés et leur articulation par l'action de quelques médiateursclés ont constitué un levier important pour assurer ensuite cette diffusion de la notion de SE et du cadre d'analyse du MA dans des espaces différents. Dans une dernière section, nous avons cherché à repérer les stratégies d'internationalisation mais aussi, en retour, les ancrages dans les espaces nationaux qui constituent des ressources souvent importantes dans la construction de la configuration épistémique du MA et de ses sousréseaux.

\section{Le Millennium Ecosystem Assessement : une gouvernance entre science et politique}

Cette première section permet de caractériser les grandes étapes du processus du MA. On distingue en particulier la phase préparatoire (1998-2000), le lancement (2000-2001), le déroulement proprement dit du MA (2001-2005) et ses suites (depuis 2005). L'entrée chronologique est complétée par une description des modalités principales de pilotage et de gouvernance à chaque étape du processus.

\section{Les premières étapes du MA et sa structure organisationnelle}

Peu de temps après la création de la Convention sur la diversité biologique (CDB) en 1992, une initiative d'envergure est prise pour cerner les enjeux de la biodiversité à l'échelle de la planète. L'exercice du Global Biodiversity Assessment (GBA), initié par le PNUE et soutenu par le Global Environment Facility (GEF) [19931995] se veut un exercice scientifique indépendant pour dresser l'état de l'art sur les multiples questions liées au sujet complexe de la biodiversité (Heywood, 1995). Ce rapport ne formule pas de recommandations aux décideurs et véhicule le message principal que les connaissances des processus liés aux questions de la diversité biologique sont encore très limitées. Selon R.T. Watson, qui a présidé ce premier exercice d'évaluation internationale de la biodiversité, le GBA a été un excellent travail scientifique révisé par des experts, mais il n'a pratiquement pas eu d'impact sur la formulation des politiques car il a été conduit comme un exercice non gouvernemental, sans appropriation par les pouvoirs publics (Watson, 2005).

L'idée d'une évaluation globale des écosystèmes est alors lancée en mai 1998 à l'occasion d'une réunion de brainstorming organisée par le World Resources Institute (WRI), un think tank américain. Le WRI publie depuis 1986 un rapport sur l'état des ressources dans le monde (World Resources), en collaboration avec le PNUE, le PNUD et la Banque mondiale.

Dans un premier temps, un comité de pilotage exploratoire (Exploratory Steering Committee) est constitué et se réunit deux fois, en février et en octobre 1999. Il comprend 33 personnes, dont une quinzaine de représentants du monde scientifique (universités, centres internationaux de recherche), une douzaine de représentants $\mathrm{d}$ 'organisations internationales (Onu, Banque mondiale, $\mathrm{FAO}$, etc.) ou d'administrations nationales (Norvège, Chine, Slovaquie, Colombie), un représentant du WRI, de l'UICN ${ }^{2}$ et du WBCSD $^{3}$ (secteur privé). Cette diversité d'appartenance institutionnelle reflète la volonté de construire un processus multiacteurs.

Ce comité de pilotage exploratoire est ensuite remplacé par un conseil d'administration (the MA Board of Directors ou Board) qui tient sa première réunion en juillet 2000. Cette instance se compose de 49 personnes au total (20 institutionnels et le reste à titre personnel). Il est coprésidé par R.T. Watson et A.H Zakhri. Le démarrage du MA est effectif début 2001. Le MA reprend la structure de fonctionnement du Giec en trois groupes de travail sur les tendances, les scénarios et les implications

\footnotetext{
Union internationale pour la conservation de la nature.

3 World Business Council for Sustainable Development.
} 
politiques ${ }^{4}$. Un quatrième groupe est constitué pour suivre la réalisation d'évaluations régionales des écosystèmes. Une instance de pilotage opérationnelle est mise en place : le Panel d'évaluation des écosystèmes (Ecosystem Assessment Panel), composé des présidents des différents groupes de travail et de quelques personnes ressources. Cette instance est considérée comme l'instance opérationnelle du MA. Une petite équipe technique, dirigée par W. Reid, coordonne les activités dont la conduite est assurée par plusieurs agences d'exécution dont les missions sont régies par des termes de références contractés avec le PNUE ${ }^{5}$.

La structure de gouvernance du MA distingue donc l'instance politique (Board) des instances opérationnelles chargées de l'évaluation scientifique (Panel d'évaluation et agences d'exécution) ${ }^{6}$. On verra dans les sections suivantes que cette apparente séparation entre le scientifique et le politique ne reflète que très partiellement la nature au contraire très imbriquée du processus, avec des articulations étroites entre scientifiques et décideurs, à la fois par des mécanismes organisationnels, mais aussi et surtout du fait de la trajectoire des personnes impliquées de près dans la conduite du MA.

\section{Le déroulement du MA et ses suites}

Le déroulement du MA proprement dit va s'échelonner entre février 2001 et mars 2005. Le Panel d'évaluation a joué un rôle-clé dans le cadrage général du travail, en coordination étroite avec le Board, qui se traduit en particulier par la publication du cadre d'analyse du MA en 2003 (Millennium Ecosystem Assessment, 2003).

Plusieurs documents jalonnent le processus du MA. Le rapport d'évaluation, considéré comme le produit principal du MA, a été publié en 2005 sous forme de trois volumes, reprenant les résultats des groupes de travail : (1) l'état actuel et les tendances des écosystèmes (2), les scénarios et (3) les réponses politiques. Chacun des volumes a été construit sous la responsabilité d'un

4 Le Giec est à l'époque, comme aujourd'hui, considéré comme un modèle $\mathrm{d}$ 'interface entre science et décision. R. Watson, qui a présidé le MA, a présidé aussi le Giec pendant la même période (de 1997 à 2002). Plusieurs autres experts de premier plan du MA sont aussi impliqués dans le Giec: H. Gitay, R. Leemans, I. Baste, J. Sarukhan, etc. facilitant ainsi les apprentissages entre ces différents exercices.

5 En effet, une partie des financements (comme ceux du GEF/Banque mondiale) devaient transiter par une agence des Nations unies, ici le PNUE, qui a ensuite sous-contracté avec sept agences d'exécution localisées sur plusieurs continents : ce caractère multisitué du secrétariat du MA semble avoir concouru à un élargissement de la diffusion de ses résultats.

6 Le lecteur trouvera le détail de la composition de ces instances sur le site du MA : http:/ / www.maweb.org. groupe de travail ad hoc. Chaque chapitre a été rédigé sous la responsabilité d'un ou de quelques coordinateurs animant une équipe d'auteurs principaux, de contributeurs et d'éditeurs. Une deuxième série de rapports a été produite pour valoriser des études conduites à des échelons locaux ou à des niveaux géographiques intermédiaires : au total, 18 évaluations sous-globales (subglobal assessments) sont publiées et 15 études complémentaires. Enfin, des documents de synthèse ont été rédigés. Une synthèse globale tout d'abord (Millennium Ecosystem Assessment, 2005), accompagnée par la suite de cinq synthèses thématiques, chacune visant une audience spécifique: la Convention sur la diversité biologique (CDB), la Convention des Nations unies sur la lutte contre la désertification (UNCCD), la convention de Ramsar (zones humides), le secteur privé et le secteur de la santé.

$\mathrm{Au}$ total, le processus aura mobilisé plus de 1300 experts pendant plus de quatre ans, à la fois pour contribuer à la rédaction des documents produits par l'évaluation, mais aussi pour leur relecture qui associe largement des représentants de gouvernements, des organisations et des conventions internationales. Une analyse plus fine de ces experts permet de mettre à jour la dynamique de mobilisation des connaissances et des réseaux qui est à l'origine de l'écho rencontré par le MA après sa publication, et des effets qu'il produira par la suite.

\section{Connecter des mondes entre eux : vers la construction d'une vision commune?}

Parmi l'ensemble large des experts impliqués dans le MA, on peut identifier un noyau plus resserré composé d'un nombre limité d'individus, qui sont parvenus à connecter des sous-réseaux relativement disjoints. En assurant une fonction de médiation entre des milieux professionnels et institutionnels très différents, quelques individus-clés du processus du MA vont progressivement construire une configuration associant plusieurs sous-réseaux. Notre hypothèse exploratoire est que le caractère composite et ramifié de cette configuration est, en partie, à l'origine de la diffusion large et diversifiée des conclusions du MA et de son cadre d'analyse.

\section{Quatre sous-réseaux principaux}

Dans un premier temps, afin de restreindre l'univers d'étude de ces experts et scientifiques du MA, nous avons construit une base de données des individus impliqués dans le processus. Nous avons procédé par agrégations successives de groupes de personnes en commençant par intégrer de façon exhaustive les 
participants des instances formelles du MA : le comité exploratoire ( 31 individus), le Board (48 individus), le panel d'évaluation (14 individus). Nous sommes ensuite parti des principaux produits du MA et, pour chacun d'eux, nous avons identifié les responsables de volumes ou de rapports complets. En général, les individus ayant eu des responsabilités éditoriales ont souvent aussi participé directement à la rédaction de certains chapitres. Cette première sélection permet de constituer un ensemble d'environ 120 personnes ayant eu plusieurs responsabilités éditoriales et/ou politiques dans le processus du MA. Nous avons ensuite analysé plus en détail cet ensemble de 120 personnes grâce à des enquêtes qualitatives basées sur une analyse des trajectoires de ces experts (sur la base de leur CV ou d'informations biographiques diverses), sur des entretiens semi-directifs effectués auprès des acteurs-clés du processus et sur un travail d'analyses des archives du MA. Cette démarche d'enquête a permis de repérer au moins quatre sousréseaux.

Un premier sous-réseau rassemble des scientifiques (écologues, biologistes, économistes) s'inscrivant plus ou moins directement dans l'émergence de la discipline récente de l'économie écologique (Ecological Economics). Si on devait résumer en une phrase l'esprit de ce sousréseau, on pourrait dire «valoriser les écosystèmes pour alerter les décideurs ». Il puise ses racines dans la dimension scientifique de la notion de SE, née de la rencontre entre des écologues, des biologistes et des économistes de l'environnement, mais qui, comme la notion de biodiversité, a été forgée aussi dans l'optique d'alerter les décideurs sur l'urgence écologique (Takacs, 1996). Les travaux de P. Ehrlich et H. Mooney, G. Daily et ses collaborateurs de Stanford s'intéressent de longue date aux SE. En économie, c'est dans le courant de l'Ecological Economics que va fortement se développer la notion de SE, notamment par l'intermédiaire d'auteurs-clés tels que R. Costanza et R. de Groot (Méral, 2012). Dès le début des années 1990, le Beijer Institute de Stockholm va être le lieu de rencontres de scientifiques de divers horizons dans des programmes de recherche sur l'environnement, et plus spécifiquement sur les SE. Au moins quatre membres du Panel d'évaluation du MA ont été impliqués à un moment ou un autre dans les activités du Beijer Institute : P. Dasgupta, K. Chopra, S. Carpenter et R. Hassan. Aujourd'hui, le Beijer Institute met en avant ses relations étroites avec les communautés scientifiques de l'écologie et de l'économie : plusieurs écologues américains de renom en sont membres comme S. Carpenter, S. Chapin, P. Ehrlich, C.S. Holling, S. Levin, J. Lubchenco. Le département de biologie de l'université de Stanford est un autre lieu-clé du processus d'émergence, puis de diffusion des idées autour de la notion de SE.

Un deuxième sous-réseau se structure autour de deux sociétés savantes internationales: l'ICSU et
Diversitas ${ }^{7}$. On pourrait le qualifier de « pour une écologie globale intégrée aux sciences du système Terre », car il puise ses racines dans la construction progressive de larges programmes pluridisciplinaires mondiaux visant la compréhension des processus affectant la biosphère. Les prémices de ce sous-réseau sont à rattacher en particulier au lancement à la fin des années 1980 d'un programme international de recherches visant à construire un cadre d'analyse englobant des transformations subies par la Terre, 1'IGBP (International GeosphereBiosphere Programme) dont un des programmes associés est centré sur les liens entre écosystèmes terrestres et changement globaux, le GCTE (Global Change and Terrestrial Ecosystems) [Kwa, 2005]. La particularité des personnes impliquées dans ces programmes est de chercher à articuler l'analyse des écosystèmes avec la problématique du changement climatique, très en vogue au niveau international. C'est cette approche systémique qui est développée au sein du MA et qui va permettre $\mathrm{d}^{\prime}$ asseoir la notion de SE sur un socle de recherches plus larges, connecté aux problématiques de changement global. Les programmes de recherches lancés et labellisés par l'ICSU et Diversitas constituent de véritables canaux de recrutement d'experts scientifiques de forte crédibilité scientifique pour le MA, tout en facilitant la diffusion rapide des idées dans les sphères scientifiques internationales.

Un troisième sous-réseau peut être identifié avec les conventions internationales et leurs arènes scientifiques, élargies aux organisations internationales de l'environnement (PNUE). On pourrait le qualifier de "seuls légitimes au niveau international sur les questions environnementales ». La principale convention concernée est la Convention sur la diversité biologique et son organe scientifique et technique, le SBSTTA (Subsidiary Body on Scientific, Technical and Technological Advice), mais ce sous-réseau implique aussi des représentants d'autres conventions internationales (Ramsar, $\mathrm{CITES}^{8}$, etc.). Des représentants de ces organisations internationales onusiennes sont étroitement associés au pilotage du processus du MA : déjà bien représentées au Board, elles sont associées au comité exécutif qui le pilote plus étroitement avec plusieurs réunions annuelles ${ }^{9}$. Une partie des scientifiques et experts mobilisés dans ce processus ont des profils hybrides combinant une forte renommée

\footnotetext{
7 International Council of Science (ICSU). Diversitas est un programme lancé en 1992 par ICSU en collaboration avec l'Unesco et l'International Union of Biological Sciences (IUBS), elle-même membre de l'ICSU.

8 Convention sur le commerce international des espèces de faune et de flore sauvages menacées d'extinction.

9 Le comité exécutif du Board du MA se compose de représentants de CDB, CCD, Ramsar, PNUE, GEF, et des présidents des autres instances organisationnelles du MA.
} 
scientifique avec une carrière internationale au sein de la $\mathrm{CDB}$, soit comme secrétaire exécutif (A. Cropper, C. Juma, H. Zedan) ou comme président de son SBSTTA (P. Schei, A.H. Zakri, C. Samper, A. Oteng-Yeboah, C. Prip). Ils ont été surtout impliqués dans la phase de préparation du MA (1998-2000) à travers leur participation à son comité exploratoire, et/ou dans son instance politique, le Board. En effet, dans l'esprit des promoteurs du $\mathrm{MA}^{10}$, l'implication des conventions internationales et des organisations intergouvernementales devait permettre par extension de sensibiliser les gouvernements et $d$ 'influencer les politiques publiques. Parmi ces experts, A. Cropper et A.H. Zakri joueront un rôle de premier plan dans le processus du MA et ses suites. Après 2005, les institutions internationales comme le PNUE et la CDB vont constituer des supports importants du travail d'influence que les promoteurs du MA vont déployer. Les conventions internationales constituent sans aucun doute le support principal de la légitimité recherchée par le MA. On soulignera toutefois la recherche d'une indépendance mesurée à l'égard de ces organisations internationales. En effet, tout en obtenant les soutiens financiers et politiques nécessaires à la légitimité politique du processus, les organisateurs du MA ont cherché à préserver son indépendance de fonctionnement, en particulier à l'égard du PNUE qui aurait aimé avoir plus de contrôle sur la gestion du processus ${ }^{11}$.

Le quatrième sous-réseau est composé de scientifiques et d'experts de la Banque mondiale et de centres internationaux de recherche affiliés au CGIAR (Consultative Group of International Agricultural Research) et plus globalement du monde du développement. On pourrait qualifier ce sous-réseau de « le développement, c'est nous ! » Comme les trois premiers, il est relativement composite, mais met en avant cette dimension de développement. D'un côté, la Banque mondiale se positionne, comme souvent, en institution hégémonique sur les questions de développement et, plus récemment, sur la mise en œuvre des politiques environnementales, avec une action plus controversée dans ce domaine (Goldman, 2001 ; Young, 2002). L'implication de cette institution doit beaucoup à R.T. Watson qui y occupe depuis 1996 le poste de responsable du département Environnement. La Banque mondiale a joué un rôle

\footnotetext{
10 Parl'expression « promoteurs » du MA, on entend le « premier cercle » des quelques personnes assurant des fonctions de présidence ou de direction des instances organisationnelles du MA. Voir plus loin quelques éléments de biographie des personnes concernées.

11 Cette « distance » a été instituée par la mise en place d'un secrétariat du MA multisitué : son siège est en Malaisie mais certaines activités sont pilotées par des agences d'exécution basées sur plusieurs continents. Initialement, le PNUE revendiquait fortement l'hébergement du secrétariat du MA (entretien avec W. Reid, avril 2012).
}

d'impulsion avec son rapport de 1998, Protecting our Planet, Securing our Future: Linkages Among Global Environmental Issues and Humans Needs. Un autre expert de la Banque mondiale, S. Pagiola, est associé au processus d'élaboration du cadre d'analyse du MA: c'est un promoteur actif des instruments de type "paiements pour services environnementaux», qui sont une des formes d'opérationnalisation de la notion de service écosystémique. L'implication de la Banque mondiale se traduit aussi par le financement du processus : elle contribue, à travers le GEF et des financements propres, à près de la moitié du budget total du MA. L'autre branche de ce sous-réseau est constituée par des experts et scientifiques issus des centres de recherches internationaux regroupés dans le CGIAR. Plusieurs de ses représentants participent de près au processus: D. Capistrano $\left(\right.$ CIFOR $\left.^{12}\right)$, P. Pingali (CYMMIT $\left.{ }^{13}\right)$. Le WorldFish Center de Penang (Malaisie) accueille le secrétariat technique du MA. Ces multiples ancrages dans les organisations internationales dédiées au développement et à la recherche pour le développement viennent compléter l'ancrage principal des questions de biodiversité habituellement associées aux questions de conservation des zones forestières dans les zones tropicales humides.

\section{Circulation des idées, connexion des réseaux et travail de médiation}

Les quatre sous-réseaux identifiés ne sont pas étanches ni forcément très homogènes. Ils constituent cependant des dominantes dans les trajectoires des experts et scientifiques qui peuvent cependant, au long de leur carrière, évoluer d'une institution à l'autre, contribuant à tisser des passerelles et à faciliter les échanges d'idées et parfois de ressources entre ces différents «mondes ». Les connexions entre ces quatre sous-réseaux sont le fait d'acteurs expérimentés, multipositionnés et qui constituent les piliers du travail de production et de diffusion des idées du MA dans différents milieux professionnels et espaces sociaux (R.T. Watson, W. Reid, H. Mooney, A. Cropper, H. Zakhri). Une brève présentation biographique de quelques-uns de ces acteurs permet de mieux saisir ces processus de passage et de valorisation de ressources accumulées dans un domaine.

R.T. Watson, de nationalité britannique, a une formation de chimiste, spécialiste des questions d'ozone. Il commence sa carrière avec la NASA ${ }^{14}$ pour y devenir en 1987 responsable d'un service de recherches sur l'atmosphère, puis directeur d'un programme de recherches dans la division des sciences de la terre et leurs

\footnotetext{
12 Center for International Forestry Research.

13 Centro internacional de mejoramiento de maíz y trigo.

14 National Aeronautics and Space Administration.
} 
applications en 1990. Depuis 1991, il préside le panel de conseil scientifique et technique du GEF et copréside deux groupes de travail du Giec. Il copréside aussi le GBA (1993-1995) $)^{15}$ et intègre la Banque mondiale de 1996 à 2001 où il dirigera le département environnement, avant de devenir scientifique en chef et conseiller pour le développement durable de 2001 à 2007. Il coprésidera le Board du MA (1998-2005), puis celui de l'IIASTD ${ }^{16}$ (20052007). Il a aussi été l'un des conseillers du président Clinton à la Maison-Blanche à la fin des années 1990. Depuis 2007, il est revenu dans son pays d'origine, le Royaume-Uni, où il occupe un poste de conseiller scientifique en chef du département Environnement. Il est également un des vice-présidents du bureau d'IPBES.

W. Reid, de nationalité américaine, a une formation de zoologiste complétée par une formation en économie de l'environnement. Il intègre rapidement le World Resources Institute, reconnu sur les questions environnementales aux États-Unis et proche des démocrates. Il y occupe de 1992 à 1998 le poste de vice-président chargé des programmes de recherche. En 1998, il va coordonner la préparation de la mise en place du MA dont il assurera la direction des équipes techniques jusqu'en 2005. Il a été professeur associé à l'université de Stanford (2005-2006) et dirige depuis 2007 le programme Science et conservation de la Fondation David et Lucile Packard qui a apporté son soutien financier au processus du MA.

H. Mooney, de nationalité américaine, est né en 1932. Il est le contemporain de P.R. Ehrlich, tous deux du département de biologie de l'université de Stanford, mais aussi d'E.O. Wilson (né en 1929) qui sera le père de l'expression «biodiversité » (1986). Spécialiste des questions d'espèces invasives, il a coordonné depuis la fin des années 1970 plusieurs programmes scientifiques internationaux. Il a assumé plusieurs responsabilités scientifiques : président du comité étatsunien pour le projet IGBP $^{17}$ (1986-1988), président de la Société américaine d'écologie (1988-1989), vice-président puis secrétaire général d'ICSU (1993 à 1998), il a participé au Global Biodiversity Assessment de 1993 à 1995. Il copréside avec

15 R.T. Watson dégage les enseignements de ces expériences dans un article insistant sur l'importance des processus dans ces évaluations globales (Watson, 2005).

16 International Assessment of Agricultural Knowledge, Science and Technology for Development.

17 L'International Geosphere-Biosphere Programme (IGBP) qui a débuté en 1986 était considéré comme un complément du World Researche Climate Programme (WRCP). L'objectif de l'IGBP était: " To describe and understand the interactive physical, chemical and biological processes that regulate the total Earth system, the unique environment that it provides for life, the changes that are occurring in this system, and the manner in which they are influenced by human activities. » Doté d'un secrétariat basé en Suède, l'IGBP est une initiative de l'ICSU (Kwa, 2005).
A. Cropper l'Assessment Panel du MA entre 2001 et 2005. Il devient président de Diversitas en 2007.

A. Cropper, diplômée en économie et droit international, originaire de Trinidad et Tobago, a une carrière politique nationale et internationale très dense. Sénatrice dans son pays, elle a eu des responsabilités importantes dans plusieurs organisations internationales : secrétaire exécutif de la CDB (1992-1995), elle a aussi travaillé pour la Banque mondiale, le PNUD et l'UICN. Depuis 2007, elle est directrice générale adjointe du PNUE.

Ces quelques personnes sont des acteurs-clés du processus, chacun possédant une expérience et un ancrage institutionnel lui ouvrant des réseaux diversifiés : réseaux scientifiques, réseaux politiques ancrés dans plusieurs pays ou régions (États-Unis, Malaisie/Asie, Caraïbes, etc.), réseaux internationaux liés aux conventions et organisations internationales.

Reste la question de la nature de l'implication des ONG et du secteur privé dans le processus du MA. À l'évidence, ces acteurs sont parties prenantes du processus, dès son commencement. Il semblerait toutefois que le secteur privé, comme les $\mathrm{ONG}$, a peu participé à la production intellectuelle collective du MA, mais a plutôt été associé au Board, dans une optique d'améliorer les produits du MA en fonction des attentes des «utilisateurs » qu'ils sont. Les réseaux tissés avec le secteur privé et les ONG joueront aussi un rôle très actif dans l'appropriation et la diffusion des idées produites par le MA après 2005.

\section{Réseaux transnationaux, influences nationales et construction d'un universel}

Dans les analyses biographiques réalisées, nous avons cherché à repérer les stratégies d'internationalisation mais aussi, en retour, les ancrages dans les espaces nationaux qui constituent des ressources souvent importantes dans la construction de la configuration épistémique du MA et de ses sous-réseaux. Comme le soulignent plusieurs travaux sur les élites internationalisées, nous estimons que « le national et l'international, loin de constituer l'opposition consacrée par les débats sur la mondialisation, sont étroitement imbriqués dans ces stratégies de reproduction élitistes. Dans l'espace des pratiques internationales, les opérateurs dominants sont ceux qui peuvent mobiliser des ressources acquises et homologuées dans des champs nationaux de pouvoir, en particulier des titres et des diplômes d'État. En contrepartie, la mobilisation d'un capital international de compétences et de relations représente un atout non négligeable dans les stratégies de pouvoir dans le champ national » (Dezalay, 2004; Dezalay et Rask Madsen, 2009). 
En premier lieu, on peut penser que les États-Unis ont joué un rôle important dans le processus du MA, ne serait-ce que dans l'impulsion initiale, largement liée à des acteurs nord-américains. Ce rôle des États-Unis peut s'apprécier d'abord par les acteurs impliqués dans le MA : sur la centaine d'acteurs identifiés comme ayant joué un rôle significatif dans le MA, environ un tiers sont nord-américains ou travaillent aux États-Unis. Il ne s'agit pas pour autant de défendre l'idée que les États-Unis, en tant qu'État, ont cherché à avoir une influence directe sur le processus du MA, mais plutôt de constater la présence significative de scientifiques et d'experts nordaméricains dans le MA, et, d'une manière plus indirecte, les nombreux liens avec les milieux nord-américains pouvant exister dans les trajectoires biographiques de certains scientifiques de pays en développement. En effet, les carrières internationales de scientifiques ou de hauts fonctionnaires des pays en développement comportent souvent des jalons importants que sont les diplômes et séjours scientifiques dans les universités des États-Unis. C'est le cas par exemple de C.Samper, membre du Panel d'évaluation du MA, à la double nationalité colombienne et américaine : il obtient master et doctorat à Harvard. Les neuf premières années de sa carrière se déroulent en Colombie où il aura des responsabilités scientifiques, mais aussi politiques (il représente son pays au SBSTTA de la CDB qu'il préside de 1999 à 2001), puis sa carrière se déploie aux États-Unis à compter de 2001 où il s'investit dans le Smithsonian's Museum qu'il dirigera à partir de 2008. Un autre membre de l'Assessment Panel, D. Capistrano, de nationalité philippine, après des études dans son pays, obtient un doctorat à l'université de Floride puis travaille pour les fondations Mac Arthur, puis Ford, avant de rejoindre le CIFOR en 2003. On pourrait multiplier les exemples qui montrent le rôle important joué par le champ scientifique nord-américain dans la construction des carrières internationales. Une autre modalité de ces relations multiformes avec les États-Unis est la participation de nombreux scientifiques européens ou des pays en développement aux sociétés savantes nord-américaines et principalement, dans notre cas, à l'Ecological Society of America (ESA). Plusieurs membres du Panel d'évaluation y participent (R. Scholes - Afrique du Sud, R. Leemans - Pays-Bas) et la branche latino-américaine de l'ESA joue un rôle important dans la circulation des idées sur l'ensemble du continent américain. De même, l'ESA a joué un rôle important au tournant des années 1980-1990 pour stimuler et orienter les travaux de recherche en écologie vers des problématiques concernant les écosystèmes et le changement global. En 1988, sous la présidence $\mathrm{d}^{\prime} \mathrm{H}$. Mooney ${ }^{18}, \mathrm{l}^{\prime} \mathrm{ESA}$ entreprend un travail de synthèse sur l'état des recherches en écologie

18 H. Mooney a présidé l'ESA entre 1988 et 1989. afin de définir les grandes orientations de cette discipline pour les années à venir (Lubchenco et al., 1991).

D'une manière plus générale, les États-Unis sont en pointe sur la construction progressive d'un agenda international de recherche sur les questions du changement global, dont les prises de position de l'ESA en 1991 ne constituent qu'une étape. Au milieu des années 1980, des physiciens et chimistes de l'atmosphère conçoivent, en dialogue avec quelques écologues, les bases du programme de recherche international, $\mathrm{l}^{\prime} \mathrm{IGBP}{ }^{19}$. Entre 1984 et 1988, le comité étatsunien de l'IGBP va élaborer les grandes lignes de ce nouveau programme international qui sera porté par l'ICSU, et aura son siège à Stockholm. Sous l'égide de l'IGBP, des programmes de recherche internationaux plus ciblés, comme le GCTE (Global Change and Terrestrial Ecosystems), amorcé en 1992, constitueront des étapes importantes à la fois dans la construction des connaissances sur les relations entre biodiversité, fonctionnement des écosystèmes et changement global, mais aussi dans la construction de réseaux scientifiques de plus en plus articulés entre l'écologie et les sciences de la terre (Mooney, 1998, 1999). Il faut signaler ici la nature hybride de ces programmes internationaux dont la légitimité et la crédibilité scientifiques se fondent en grande partie sur leur caractère international, tout en dépendant exclusivement des engagements financiers directs des gouvernements à travers les agences nationales finançant la recherche.

Aux États-Unis, les différents comités scientifiques de l'Académie nationale des sciences (NAS) jouent un rôle-clé dans cette interface entre science et politique. Des scientifiques comme H.A Mooney, S.Carpenter, J. Lubchenco ont eu des responsabilités importantes dans ces instances, facilitant les articulations entre programmation et financement de la recherche.

On pourrait multiplier les exemples explicitant le rôle joué par les États-Unis dans la construction des agendas de recherche internationaux. Il faut tout de même s'interroger sur la nature et les modalités de ce rôle qui ne reflète pas forcément un engagement clair de l'administration américaine. Il s'agit plutôt d'une action combinée, mais pas toujours coordonnée - car sujette aussi aux changements politiques aux États-Unis -, d'un grand nombre d'acteurs nord-américains. Selon H.A. Mooney, le gouvernement des États-Unis n'a pas beaucoup soutenu directement le processus du $\mathrm{MA}^{20}$. À l'image des travaux d'historiens américains montrant les articulations entre la lecture de la mondialisation et l'histoire propre des États-Unis (Saunier, 2004 ; Thelen, 1999), on pourrait se demander s'il ne faut pas aussi considérer ces

\footnotetext{
19 Sur l'origine et l'objectif de l'IGPB, voir la note de bas de page $n^{\circ} 17$.

20 Entretien avec H. Mooney, mars 2011.
} 
multiples formes d'engagement comme la construction d'un universel centré implicitement sur des visions et valeurs produites et partagées principalement aux ÉtatsUnis, et sans doute plus largement par des pays «riches ${ }^{21}$.

\section{Conclusion}

Cet article a permis de montrer en quoi le processus du MA en tant que tel a contribué, par sa conception et son déroulement, à faciliter l'adoption puis la large diffusion d'un nouveau cadre d'analyse, dont la notion de service écosystémique constitue le pivot. La diversité des réseaux d'acteurs mobilisés, leur articulation par l'action de quelques médiateurs-clés a constitué un levier important pour assurer la diffusion de la notion de SE dans des espaces, institutions et mondes professionnels différents. Plusieurs concepts tentent de cerner ces dynamiques en réseaux et leur rôle dans la structuration des débats et processus politiques internationaux : la notion de communauté épistémique (Haas, 1992), que nous avons légèrement adaptée en utilisant la notion de configuration épistémique, plus ouverte (Pesche et al., 2012), ou alors la notion de réseaux distribués (Cash et Clark, 2001), qui cherche à capter ces dynamiques en réseau qui traversent les frontières institutionnelles et nationales.

On peut cependant s'interroger sur le caractère transnational de ce processus et souligner que, si ces ramifications s'étendent dans de multiples directions ${ }^{22}$, les liens avec le champ scientifique aux États-Unis sont si nombreux qu'on doit s'interroger sur la constitution nécessairement hybride de ces réseaux qui, à l'image des grandes fondations américaines, "conçoivent leur action non seulement à l'intérieur de leur espace domestique, mais aussi vers le genre humain tout entier, for the welfare of the mankind [...] et nourrissent de véritables projets universels et universalisants » (Saunier, 2004). Ainsi, l'élaboration et la diffusion des concepts qui jouent un rôle croissant dans la définition des politiques environnementales doivent-elles être analysées en prenant en compte ces interactions multiples entre espaces

\footnotetext{
21 La grande majorité (entre 60 et $70 \%$ ) des rédacteurs et relecteurs des produits du MA sont originaires d'Amérique du Nord, d'Europe et d'Australie. Cette catégorie de pays " riches » est très discutable mais semble avoir encore beaucoup d'influence notamment dans les grandes conventions internationales, mais aussi dans beaucoup de milieux professionnels avec l'usage de sa version euphémisée par l'emploi de la dichotomie Nord/Sud. C'est aussi une thématique qui revient régulièrement questionner la légitimité des évaluations globales environnementales (Biermann, 2001).

22 Mais très peu dans l'espace français, comme le montre l'article de M. Hrabanski (2013).
}

nationaux, organisations internationales et réseaux transnationaux, étroitement articulés dans des dynamiques complexes et mouvantes.

\section{Références}

Biermann, F., 2001. Big science, small impacts in the South?: The influence of global environmental assessments on expert communities in India, Global Environmental Change, 11, 297-309.

Carpenter, S.R., Mooney, H.A., Agard, J., Capistrano, D., DeFries, R.S., Díaz, S., Dietz, T., Duraiappah, A.K., OtengYeboah, A., Pereira, H.M., Perrings, C. Reid, W.V., Sarukhan, J., Scholes, R.J., Whyte, A., 2009. Science for managing ecosystem services: Beyond the Millennium Ecosystem Assessment, Proceedings of the National Academy of Sciences of the United States of America, 106, 5, 1305-1312.

Cash, D.W., Clark, W.C., 2001. From science to policy: Assessing the assessment process. Faculty Research Working Papers Series, Cambridge (MA), John F. Kennedy School of Government, Harvard University.

Clark, W.C., Mitchell, R.B., Cash, D.W., 2006. Evaluating the influence of global environmental assessments, in Mitchell, R.B., Cash, D.W., Dickson, N.M. (Eds), Global Environmental Assessments: Information and influence, Cambridge (MA), MIT, 1-28.

Dezalay, Y., 2004. Les courtiers de l'international : héritiers cosmopolites, mercenaires de l'impérialisme et missionnaires de l'universel, Actes de la Recherche en Sciences Sociales, 151-152, 1, 4-35.

Dezalay, Y., Garth, B., 2002. La Mondialisation des guerres de palais : la restructuration du pouvoir d'État en Amérique latine, entre notables du droit et "Chicago boys », Paris, Éditions du Seuil.

Dezalay, Y., Rask Madsen, M., 2009. Espaces de pouvoir nationaux, espaces de pouvoir internationaux, in Cohen, A., Lacroix, B., Riutort, P. (Eds), Nouveau manuel de science politique, Paris, La Découverte.

Goldman, M., 2001. Constructing an environmental state: Ecogovernmentality and other transnational practices of a 'Green' World Bank, Social Problems, 48, 4, 499-523.

Gómez-Baggethun, E, Groot, R. (de), Lomas, P.L., Montes, C., 2010. The history of ecosystem services in economic theory and practice: From early notions to markets and payment schemes, Ecological Economics, 69, 1209-1218.

Haas, P.M., 1992. Introduction: Epistemic communities and international policy coordination, International Organization, 46, 1, 1-35.

Haas, P.M., 2000. International institutions and social learning in the management of global environnemental risks, Policy Studies Journal, 28, 3, 558-575.

Heywood, V.H., 1995. The Global Biodiversity Assessment, United Nations Environment Programme, Cambridge (UK), Cambridge University Press.

Hrabanski, M., 2013. Les experts scientifiques français dans le Millenium Ecosystem Assessment (2001-2005) : les raisons de leur absence, Natures Sciences Sociétés, 21, 2, 182 (http: / / dx.doi.org/10.1051/nss/2013095). 
Kwa, C., 2005. Local ecologies and global science: Discourses and strategies of the International Geosphere-Biosphere Programme, Social Studies of Science, 35, 923-950.

Leemans, R., 2008. Personal experiences with the governance of the policy-relevant IPCC and Millennium Ecosystem Assessments, Global Environmental Change, 18, 12-17.

Lévêque, C., 2007. À propos de l'évaluation des écosystèmes pour le Millénaire : l'économie de l'environnement à l'épreuve des faits, Natures Sciences Sociétés, 15, 77-80.

Lubchenco, J., Olson, A.M., Brubaker, L.B., Carpenter, S.R., Holland, M.M., Hubbell, S.P., Levin, S.A., MacMahon, J.A., Matson, P.A., Melillo, J.M., Mooney, H.A., Peterson, C.H., Pulliam, H.R., Real, L.A., Regal, P.J., Risser, P.G., 1991. The Sustainable Biosphere Initiative: An ecological research agenda. A report from the Ecological Society of America, Ecology, 72, 2, 371-412.

Méral, P., 2012. Le concept de service écosystémique en économie : origine et tendances récentes, Natures Sciences Sociétés, 20, 1-13.

Millennium Ecosystem Assessment, 2003. Ecosystems and Human Well-being: A Framework for Assessment, Washington, Island Press.

Millennium Ecosystem Assessment, 2005. Ecosystems and Human Well-being: Synthesis, Washington, Island Press.

Mooney, H.A., 1998. The Globalization of Ecological Thought, Oldendorf, Germany, Ecology Institute.

Mooney, H.A., 1999. On the road to global ecology, Annual Review of Energy and the Environment, 24, 1-31.
Norgaard, R.B., 2008. Finding hope in the Millennium Ecosystem Assessment, Conservation Biology, 22, 4, 862-869.

Norgaard, R.B., 2010. Ecosystem services: From eye-opening metaphor to complexity blinder, Ecological Economics, 69, 1, 1219-1227.

Pesche, D., Méral, P., Hrabanski, M., Bonnin, M., 2012. Ecosystem services and payments for environmental services: Two sides of the same coin?, in Muradian, R., Rival, L. (Eds), Governing the Provision of Environmental Services, Dordrecht, Nederland, Springer.

Saunier, P.-Y., 2004. Circulations, connexions et espaces transnationaux, Genèse, 57, 110-126.

Stephens, J.C., Hansson, A., Liu, Y., Coninck, H. (de), Vajjhala, S., 2011. Characterizing the international carbon capture and storage community, Global Environmental Change, 21, 2, 379-390.

Takacs, D., 1996. The Idea of Biodiversity: Philosophies of Paradise, Baltimore, London, The Johns Hopkins University Press.

Thelen, D., 1999. The nation and beyond: Transnational perspectives on United States history, The Journal of American History, 86, 965-975.

Watson, R.T., 2005. Turning science into policy: Challenges and experiences from the science-policy interface, Philosophical Transactions of the Royal Society B: Biological Sciences, 360, 1454, 471-477.

Young, Z., 2002. A New Green Order: The World Bank and the Politics of the Global Environment Facility, London, Sterling (VA), Pluto Press.

Reçu le 18 juin 2012. Accepté le 21 novembre 2012. 